\title{
Entrenamiento estratégico en la enseñanza de la comprensión lectora del inglés como lengua extranjera. Las estrategias textual y discursiva
}

\author{
Piedad Fernández Toledo \\ Universidad de Murcia
}

Recibido: 11 marzo 2005 / Aceptado: 4 abril 2005

ISSN: $1697-7467$

\begin{abstract}
This paper presents an overview of strategic knowledge as a component of the reader's prior knowledge and its effect on reading comprehension. Different works which show the benefits of applying strategic knowledge to the reading process are revised, to focus on studies in second and foreign language reading comprehension. These show a positive effect of overt instruction on the use of reading strategies, especially those related to text structuring and the recognition of rhetorical functions (rhetorical strategies). Implications point to the advantages of explicit instruction and contrastive approaches in dealing with reading comprehension strategies in foreign and second language classrooms.
\end{abstract}

Key words: reading comprehension; prior knowledge; structural strategy; rhetorical strategy.

RESUMEN: Se presenta una revisión del conocimiento estratégico como componente del conocimiento previo y su efecto sobre la comprensión lectora, que parece ser positivo especialmente en el aprendizaje de segundas lenguas y de lenguas extranjeras. Otros estudios apuntan al beneficio del entrenamiento estratégico explícito, especialmente en el uso de estrategias relacionadas con la estructuración textual y con el reconocimiento de la función retórica (estrategias de género o retóricas). Se concluye sobre las ventajas de una instrucción explícita y del uso de enfoques contrastivos en la enseñanza de estrategias de comprensión lectora en las aulas de lenguas extranjeras y de segundas lenguas.

Palabras clave: comprensión lectora; conocimiento previo; estrategia estructural; estrategia retórica.

\section{INTRODUCCIÓN}

La comprensión lectora de textos en inglés supone un paso obligatorio para lograr una alfabetización académica plena. La lengua inglesa es, hoy por hoy, el principal idioma de comunicación científico-académica, de manera que para lograr este objetivo se impone su manejo, al menos en lo tocante a comprensión y expresión escrita. No obstante, un hablante adulto con relativo nivel de inglés cuenta con un conocimiento previo que hace que este manejo no sea tan arduo como pudiera preverse. 
La teoría de los esquemas de conocimiento previo supone una pequeña revolución en relación con los distintos modelos de comprensión lectora, especialmente los de corte interactivo. Distintos componentes, primero teóricamente y más tarde mediante evidencia empírica, se han considerado decisivos en la interacción entre conocimiento previo y comprensión de textos escritos. Un primer componente es la experiencia previa lectora en la lengua materna. En la comprensión de una lengua extranjera hay un bagaje que se transfiere de la L1 a la L2, ${ }^{1}$ al menos en parte (Eisterhold et al., 1990; Carrell, Carson y Zhe, 1993; Tao y Healy, 1996). El segundo componente es el conocimiento previo conceptual o temático, cuya influencia en la comprensión parece ser variable dependiendo de factores como la dificultad del texto (Johnson, 1981; Alderson y Urquart, 1988; Salager-Meyer, 1991). Un tercer componente que parece tener un efecto relativo es la competencia lingüística en la lengua meta (Olah, 1984; Zuck y Zuck, 1984; Perezt y Shoham, 1990; Hammadou, 1991). Por último, cabe considerar el uso de estrategias (especialmente las de tipo metacognitivo) en el reconocimiento de patrones en los textos, así como en el procesamiento lector en general. Es éste un apartado que ha ido cobrando cada vez mayor relevancia en distintos modelos de comprensión lectora en L2 que han aparecido en la literatura.

A continuación se desglosan algunos de los trabajos, junto con los resultados más significativos, que apuntan al beneficio de la inclusión del componente estratégico en la instrucción de la comprensión lectora de textos. Nos centraremos específicamente en los textos escritos en inglés como lengua extranjera o como segunda lengua, por darse ambas situaciones en el mundo hispanohablante.

\section{USO DE ESTRATEGIAS EN LA COMPRENSIÓN LECTORA}

La tendencia creciente a considerar el factor estratégico se puede apreciar en la literatura producida en las últimas décadas dentro de las teorías psicolingüísticas - especialmente la teoría de los esquemas- y cognitivistas, así como en la investigación específicamente centrada en la comprensión lectora.

Así, en un análisis sobre los cambios en los modelos de comprensión lectora fruto de las corrientes cognitivistas, Dole, Duffy y Roehler (1991) incluyen el conocimiento estratégico como un componente de conocimiento previo en la lectura, junto con el conocimiento específico sobre el tema del texto, el conocimiento general -del mundo- sobre relaciones y estructuras sociales y el conocimiento acerca de la organización textual. Los autores definen el concepto de estrategia diferenciándolo de destrezas [skills], que habían sido el objetivo primordial en la instrucción sobre la lectura hasta esa fecha, en relación con los siguientes aspectos: intencionalidad, sofisticación cognitiva, flexibilidad y la conciencia que el lector posea de ellas (ibid: 242-243).

En este sentido, Van Dijk y Kinstch (1983) afirman que la información para el uso de estrategias específicas, como las macroestrategias, puede provenir de diversas fuentes, como pistas textuales, información contextual, memoria semántica, etc.

${ }^{1}$ Aquí y en el resto del artículo L1 se utiliza para la lengua materna y L2 tanto para la segunda lengua como para la lengua extranjera, indistintamente, en oposición a la L1. En caso de tener que diferenciarlas se utilizarán, respectivamente, las siglas SL y LE. 
Los estudios realizados sobre la comprensión lectora, tanto para las lenguas maternas como para las extranjeras, muestran una evolución desde la consideración de la centralidad del texto a la del uso de estrategias metacognitivas del aprendiz y de estrategias organizativas basadas en su estructuración (León, 1991). Esta evolución es paralela a un cambio de enfoque en los programas de enseñanza, que pasan de un énfasis en los textos a la centralidad del aprendiz y la potenciación de sus propios recursos, basándose en las teorías cognitivistas (Cooper, 1990; Poggioli, 1991; Ríos Cabrera, 1991, etc.). Así, se establece como principal objetivo en los programas de instrucción para la lectura el desarrollo de un control consciente o consciencia metacognitiva (consciencia de los mecanismos de control del propio aprendizaje y, en concreto, del proceso lector) sobre un conjunto de estrategias. Dichas estrategias abarcarían, entre otras, la determinación de importancia de las ideas del texto (en relación con su macroestructura) y el resumen de información (que requiere diferenciar la información importante y sintetizarla en un nuevo texto).

\section{ESTUdios SOBRE ESTRATEgIAS Y COMPRENSIÓN LECTORA DE SEGUNDAS LENGUAS Y LENGUAS EXTRANJERAS}

Con respecto al aprendizaje de la L2 en concreto, en las últimas décadas se comenzó a tener más en cuenta el papel de la metacognición en la comprensión lectora (Hosenfeld, 1977; Pearson Casanave, 1988; Carrell, 1988; Carrell, Carson y Zhe 1993; Kitao et. al., 1990; Manchón Ruiz, Murphy y Orts Llopis, 1993; Fitzgerald, 1995; Salataci y Akyel, 2002). Un gran número de estudios ha comparado y contrastado las estrategias de lectura en el ámbito de la L2 en particular (estudios intralingüísticos), mientras que otros han analizado y contrastado el uso de estrategias en la lengua nativa y en la lengua meta (estudios interlingüísticos).

Dentro del primer conjunto se ha averiguado que la adquisición de la habilidad lectora es evolutiva: se desarrolla conforme avanza la competencia lingüística en la L2. Los sujetos extranjeros con alta capacidad en la L2 emplean estrategias de procesamiento más cercanas a las de los nativos que los estudiantes con menor capacidad, que tienden a utilizar las mismas estrategias que en su lengua materna.

En los trabajos de carácter interlingüístico se ha observado el comportamiento de los mismos sujetos en su lengua y en la lengua meta, estudiándose la transferencia de estrategias y la interferencia de la L1 en la L2. Los resultados hasta ahora han sido complementarios y contradictorios: sigue sin estar demasiado claro hasta qué punto las estrategias de la lengua materna facilitan la adquisición o la dificultan. El estudio de Kern (1989) demuestra el efecto positivo de la enseñanza explícita en estrategias en la comprensión lectora de francés como L2 y en su habilidad inferencial. Otras implicaciones del mismo estudio se refieren a la transferencia de estrategias: el hecho de que los alumnos con más problemas de lectura en la L2 fueron los más beneficiados por la instrucción explícita puede significar que los alumnos más habilidosos transfieren más estrategias de lectura de su L1 a la clase de L2 (todos los alumnos tenían el mismo nivel lingüístico).

El estudio de Kern (1989) y otros similares ponen también en duda la noción de 'techo de competencia lingüística’ de Clarke (1988), al sugerir que una instrucción explícita y una práctica en estrategias pueden disminuir el efecto de las limitaciones lingüísticas en niveles 
bajos. El trabajo de Salataci y Akyel (2002) muestra también cómo la instrucción en estrategias para la comprensión lectora de la L2 puede mejorar también estas destrezas en la lengua materna.

McDonough (1995: 57) recoge la que posiblemente hasta esa fecha sea la lista más exhaustiva de estrategias de lectura en un ámbito contrastivo, agrupándolas en las categorías de:

1. ayudas técnicas (búsqueda de ideas principales, búsqueda de información concreta, reconocimiento de cognados, etc.);

2. de clarificación y simplificación, como uso de paráfrasis, o identificación de categorías sintácticas o gramaticales;

3. de detección de coherencia, por ej., identificación del marco general ("macroframe"), uso de conocimiento previo sobre el tema, identificación de información clave, anticipación del contenido, reconocimiento de estructura textual o integración de la información;

4. de control y verificación, como la relectura, la corrección de errores, el cuestionarse la información textual, o variación de la velocidad de lectura.

Algunos de los elementos que McDonough (1995) llama estrategias en esta lista, no son otra cosa que el uso activo y consciente de los tipos de conocimiento previo descritos hasta ahora (temático, gramatical, pragmático e incluso estratégico de carácter general, que pueden haberse utilizado en la L1). En el caso de otros, como el reconocimiento de palabras de raíz común (cognados), se trataría más bien de estrategias puramente interlingüísticas, más susceptibles de aprenderse a través de la instrucción explícita.

\subsection{Estrategias de lectura: estudios sobre hablantes bilingües}

Trabajos con hablantes bilingües como los de Cohen (1991), y Dole, Brown y Trathen (1996) sobre lectores eficaces, incluyen estrategias que se pueden agrupar dentro de alguna de las categorías recogidas en McDonough (1995). Estas hacen hincapié en el uso del contexto y del conocimiento previo, en la búsqueda de semejanzas con la L1 y en la acción correctiva. En Ridgway (1994: 230) se sugiere que tanto el co-texto como el conocimiento previo son áreas pertenecientes al contexto y se enfatiza el papel que las estrategias relacionadas con éste desempeñan en la comprensión lectora.

La investigación de Carrell (1989) con alumnos hispanohablantes y angloparlantes destaca la correlación positiva entre el comportamiento estratégico y la comprensión lectora en la SL. Además se hallan diferencias en el tipo de estrategia utilizada, que parecen estar relacionadas con el nivel de habilidad en la SL.

Dole, Brown y Trathen (1996) continúan, dentro de la misma línea, comprobando experimentalmente el uso de estrategias de lectura en estudiantes bilingües, latinos y anglosajones. Los resultados e implicaciones apuntan a que:

1. Los lectores eficaces parecen poseer una consideración unitaria de la lectura en cualquier lengua;

2. Los lectores eficaces utilizan las siguientes estrategias: 
- resolución de vocabulario desconocido (al que prestan más atención que los lectores monolingües);

- uso del contexto;

- uso del conocimiento previo relevante, relacionándolo con el texto; realización de inferencias y conclusiones sobre éstas; búsqueda de cognados; traducción;

- control de la comprensión, identificando los obstáculos;

- formulación de preguntas durante la lectura

3. En comparación con la L1, en la SL

- se recurre más al conocimiento previo que en la L1;

- además existe más control sobre la comprensión;

- se utilizan dos estrategias adicionales: traducción y búsqueda de términos con la misma raíz.

4. El ser consciente de las diferencias entre L1 y L2 parece facilitar la transferencia de estrategias.

5. En cuanto a las posibles diferencias en el comportamiento estratégico según sea la $L 1$, parece que entre los hablantes latinos el vocabulario desconocido es el principal obstáculo para la comprensión, mientras que para los anglosajones no hay necesidad de determinar el significado de vocabulario desconocido; además estos últimos invocan continuamente a su conocimiento previo extra- lingüístico.

6. Parece que el ser consciente de las relaciones entre la L1 y la SL ayuda también al éxito en la comprensión lectora; así, los lectores con menos éxito no pueden discernir el propósito de lectura. Lo que es más, parecen no saber cómo utilizar la L1 para impulsar la lectura en SL, lo cual provoca un bajo rendimiento académico.

Al igual que otros muchos autores que han estudiado aspectos más concretos de la lectura, como el reconocimiento léxico o las estrategias estructurales, ellos sugieren la necesidad de centrarse en la relación entre la L1 y la SL, y potenciar un mayor uso de la L1 que facilite la adquisición de la SL, mediante la explotación del conocimiento previo. En concreto, el utilizar la traducción, la concienciación sobre palabras con similitud formal (cognados) y la transferencia de información, son estrategias exclusivas de la lectura bilingüe que hay que aprovechar. Este estudio y posteriores (Jiménez, 1997) vuelven a demostrar, además, que el conocimiento metalingüístico supone un gran beneficio, y que la transferencia de estrategias puede compensar la falta de competencia lingüística.

\subsection{Tipologías textuales y estrategia estructural}

Una gran parte de las investigaciones sobre comprensión lectora en la L2 se ha centrado en la configuración de los textos y su impacto en la comprensión lectora por parte de hablantes extranjeros. Muchas se basan en otras llevadas a cabo en la L1 y que demostraban ya la relación entre configuración textual y procesamiento lector (Meyer 1975, 1977; Meyer y Penland, 1982), e incluso apuntaban a la estrategia estructural como la dominante, en un procesamiento de lectura predominantemente descendente (Meyer y Grice, 1982).

Connor (1984) en Carrell (1985: 729) realiza un estudio contrastivo sobre recuerdo de prosa expositiva con la estructura problema-solución por parte de hablantes nativos y nonativos, con el inglés como L2: los estudiantes de ambos grupos recordaron menos ideas de 
nivel inferior, pero el mismo número de ideas de nivel superior ${ }^{2}$. En Carrell (1984) se describe un estudio similar sobre varias tipologías textuales y su incidencia en la comprensión lectora por parte de lectores con diferentes L1s. Carrell defiende el entrenamiento metacognitivo en el aula para mejorar las destrezas de comprensión lectora, ya que la posesión de esquemas formales parece facilitar, según su estudio, el procesamiento de la organización textual.

En Carrell (1985) se demuestra asimismo que la enseñanza explícita de la organización retórica textual (de nivel superior) puede facilitar la comprensión lectora de textos en la L2, ayudando no sólo al recuerdo de ideas principales, sino también al de las secundarias. Al mismo tiempo Carrell apunta a la reacción positiva de los estudiantes ante este tipo de entrenamiento.

Tras este estudio Carrell concluye sobre la necesidad de refinar las estrategias didácticas y determinar su longitud óptima, así como de descubrir si existen efectos diferenciales en distintos niveles de competencia lingüística. Asimismo lanza una advertencia: "Teaching the prototypical patterns of different texts would be inappropiate unless such instruction occurs in conjunction with helping students, in a number of ways, to acquire meaning from text" (ibíd: : 742).

El trabajo de Lee y Riley (1990) analiza los efectos de proporcionar al lector un marco orientado retóricamente, de prelectura, como acompañante del texto para facilitar su comprensión. En su experimento se comprueba que:

1) el recuerdo de prosa altamente organizada es mayor que el de prosa menos cohesionada (la estructura problema -solución fue más fácil de recordar que la estructura serie de descripciones);

2) un marco que indique la organización retórica del texto es un adjunto textual efectivo para el recuerdo de la información textual;

3) los lectores que utilizan la organización retórica del texto leído para organizar sus recuerdos, recuerdan más que los que no la utilizan;

4) el recuerdo de unidades ideacionales de nivel superior se ve afectado por el tipo de estructura discursiva del texto leído;

5) para ciertos tipos de estructura discursiva el recuerdo de las ideas de nivel superior ("top level idea units") se ve afectado por la presencia de un marco orientado retóricamente.

Los autores comparan también el efecto de la estructura retórica en la comprensión con el de la familiarización temática, concluyendo que, al menos en este estudio, la primera es más determinante (ibíd.: 34). Así, sugieren un enfoque de la enseñanza de idiomas basado en las estructuras retóricas.

Raymond (1993) persigue igualmente averiguar si la instrucción sobre la estructura organizativa de los textos puede ayudar a la comprensión lectora de la lengua extranjera, en este caso el francés; en concreto, se mide el uso de la estrategia estructural propuesta por

${ }^{2}$ Las expresiones "nivel superior" y "nivel inferior" hacen alusión al tipo de procesamiento lingüístico, que abarcaría desde el reconocimiento de la información como discurso (nivel superior) hasta el reconocimiento de fonemas (nivel inferior). 
Meyer y Grice (1982) y su incidencia en la comprensión lectora. Se trata de una réplica del estudio de Meyer y Grice en la L1 sobre instrucción en estructuras de nivel superior y comprensión lectora. Es una réplica también de Carrell (1985), con la diferencia de que en el estudio de Carrell los hablantes son de distintas procedencias y niveles, y aquí los alumnos son 43 anglohablantes de nivel intermedio superior, con francés como LE. Además, se diferencia en que aquí los sujetos respondieron en su L1, siguiendo las recomendaciones de Lee (1986). Los resultados de su estudio indican una relación entre uso de estrategias y habilidad lectora, por un lado, y posible transferencia de estrategias de lectura de la lengua materna a la L2. Según la autora: "The use of top level sentence to organize recall, or the structure strategy, depends on subjects' prior knowledge and on text content" (ibíd.: 455). Raymond contempla la complejidad del fenómeno de la comprensión lectora, debido según ella a la influencia conjunta de componentes como estructura retórica, conocimiento temático y características del lector:

Strategic training in second language reading is a highly complex and difficult undertaking, and for a reason. It is not the singular effect of a particular strategy, as for example the structural strategy, on second language reading, but the interaction of the strategy with text content, reader interest and background knowledge, and reader perceptions of text difficulty that requires investigation. (Raymond, 1993: $455)$.

La investigación de Lahuerta (1994) se centra en aprendices de inglés con español como L1. Los objetivos de su trabajo son, por un lado, analizar el efecto de la estructura (organización retórica de prosa expositiva) en la compresión lectora de inglés como lengua extranjera, y, por otro lado, estudiar la relación entre uso de estructura y comprensión y reproducción de información. Por uso de estructura, Lahuerta entiende no sólo su reproducción, sino el reconocimiento consciente de esa estructura.

Partiendo de los resultados de su estudio, Lahuerta sostiene que, sólo cuando coinciden en el lector la reproducción y el reconocimiento consciente de la estructura textual, tiene ésta un efecto positivo sobre la comprensión lectora y en la reproducción de información presente en un texto. Por el contrario, cuando el lector no reconoce la organización textual (incluso si es capaz de reproducirla), ésta no afecta a su rendimiento en la actividad lectora, según el estudio.

Es necesario, por tanto, según la autora, hacer conscientes a los lectores acerca de la organización textual para mejorar su capacidad de comprensión lectora, y hacerlos además capaces de interpretar la información retórica de los textos. La interpretación supone utilizar tanto el conocimiento esquemático formal, como las señales textuales, buscando el significado en el texto y en las propias predicciones. A este entrenamiento en esquemas formales hay que añadir un componente procedimental a desarrollar en los lectores, de manera que se conviertan en "procesadores interactivos", seleccionando e integrando pistas de distintos niveles para llegar a una interpretación plausible de los textos. Su propuesta pedagógica, basada en estos resultados, es la de incrementar en los aprendices la concienciación sobre estos procedimientos y su trascendencia.

Un estudio similar y más reciente de Bimmel, Van der Bergh y Oostdam (2001) con alumnos holandeses de secundaria, persigue comprobar la eficacia de la instrucción explícita 
en estrategias en la comprensión lectora tanto de la lengua materna como de la lengua meta. Sin embargo, aunque se demuestra el éxito de la enseñanza explícita de estrategias concretas de lectura (búsqueda de información clave dentro del texto, la detección de marcadores estructurales, la realización de preguntas sobre el contenido y la realización de mapas semánticos) sobre la comprensión, los autores no consiguen comprobar la transferencia de estas estrategias a la lengua meta, que en este caso era el inglés.

Levine y Reves (1994) proponen un modelo de lectura en LE de carácter tetradimensional, donde la comprensión lectora global, una de las dimensiones, se vería afectada por cada uno de los esquemas propuestos por Carrell (1987): lingüísticos (destrezas de decodificación y procesamiento discursivo), de contenido (conocimiento del área de contenido del texto) y formales (reconocimiento de la estructura retórica del texto). En su experimento se intenta comprobar el efecto combinado de los tres tipos de esquema sobre la comprensión de textos académicos con diferentes estructuras retóricas, el peso relativo de cada uno de los componentes, y la influencia de actividades de inducción de esquemas sobre la comprensión. Los sujetos eran estudiantes matriculados en cursos de inglés como lengua extranjera de nivel avanzado.

Este complejo estudio, donde se realizan variadas pruebas a un total de 95 estudiantes universitarios de primer curso con inglés como LE, muestra una interacción significativa entre los distintos tipos de esquema. En general, el efecto del uso de cada esquema no es igual, teniendo más peso los de contenido, luego los lingüísticos y, por último, los formales. Esta influencia, sin embargo, parece ser diferente según el tipo de texto, teniendo los esquemas lingüísticos más peso al analizar los datos según las diferentes estructuras retóricas textuales. En los tests sobre los textos descriptivos, las preguntas relacionadas con los esquemas lingüísticos muestran una alta correlación significativa con la puntuación global; en los tests sobre los textos argumentativos y analíticos, por otro lado, la puntuación de las preguntas orientadas al uso de los tres tipos de esquema muestra una alta correlación con la puntuación global.

De esta manera el estudio confirma la contribución de los cuatro tipos de esquema, aunque no en el mismo grado: cuando los tipos de texto no se diferencian en el análisis, el conocimiento que el lector de una lengua extranjera tiene sobre el área temática del texto (esquemas de contenido) así como el conocimiento lingüístico previo (esquemas lingüísticos) parecen ser factores determinantes en la comprensión global del texto.

El peso relativo y la interacción cumulativa de los tres tipos de esquema difieren con respecto al tipo de organización textual. Los esquemas formales del lector de lenguas extranjeras, por otro lado, sólo parecen ser efectivos cuando el texto tiene una estructura argumentativa, altamente organizada. En esos textos, la habilidad del lector para seguir el argumento lógicamente estructurado tiene más peso que en los textos descriptivos, mostrando las entrevistas retrospectivas con los sujetos una tendencia similar. Estos resultados concuerdan con los de otros estudios, como el de Carrell (1985), en los que los textos con estructura más cohesionada resultan tener más peso en la comprensión.

De los diferentes trabajos reseñados hasta ahora se pueden extraer los siguientes datos:

1. La estructura textual facilita la comprensión a nivel general del mismo modo que en el caso de las lenguas maternas (Carrell, 1985; Connor, 1984).

2. La estructura textual en la L2 se percibe con distinto nivel de dificultad según sea la tipología del texto y la lengua- origen del lector (Carrell, 1984). 
3. Parece ser, según el mismo estudio y el de Levine y Reves (1994), que una organización textual más estructurada incide en un mayor nivel de comprensión; esto concuerda con las conclusiones de Demel (1990), acerca del conocimiento previo retórico y léxico como elementos que ayudan a resolver las relaciones anafóricas en los textos, facilitando su comprensión en la L2.

4. La estructura textual puede ser más determinante para la comprensión que el conocimiento de la temática (Lee y Riley, 1990) aunque esto no se confirma en el trabajo de Levine y Reves (1994), donde el conocimiento lingüístico de la L2 resulta ser el factor principal, seguido por la familiaridad temática.

5. La elaboración de los protocolos de recuerdo en la lengua materna (Raymond 1993) facilita la mayor reproducción de ideas, tanto primarias como secundarias.

6. Los trabajos de Carrell (1985), Lahuerta (1994) y Raymond (1993) demuestran la importancia de un entrenamiento metacognitivo en esquemas formales para la lectura en L2, que facilita igualmente la comprensión de ideas generales y la de información detallada.

7. El comportamiento estratégico en la L2 supone hacer uso de un procesamiento interactivo, con una utilización consciente de pistas textuales a distintos niveles según lo requiera la situación (Lahuerta 1994).

8. Parece haber transferencia de estrategias formales de lectura de la L1 a la L2 (Raymond 1993; Levine y Reves 1994).

\subsection{Estrategias discursivas o de género}

Los trabajos revisados se centran en estrategias de identificación de la estructura de los textos de forma funcional, sin incluir aspectos contextuales o pragmáticos: una estructura retórica de las propuestas por Meyer (1977), en las que se centran la mayoría de los estudios, puede aparecer en textos muy diversos (una carta, propaganda política, etc.). En la mayoría de los casos, cuando se habla de organización retórica de los textos se está aludiendo más bien a su estructuración, sin entrar en matices relativos a su finalidad pragmática. Esto lleva a que la dimensión comunicativa del texto no se llegue a contemplar en su totalidad.

Así, los textos pueden tener una estructuración similar y sin embargo cumplir una función retórica o pragmática diferente, al pertenecer a distintos géneros. Es el caso, por ejemplo, de los géneros reseña y anuncio editorial, en los cuales la macroestructura, así como las funciones textuales (descripción- evaluación, principalmente), son muy similares, pero no tanto su función comunicativa, o pragmática, o retórica (Fernández Toledo, 2002-2003). La etiqueta "retórico/-a" se ha utilizado para hacer referencia a tipologías textuales, pero no necesariamente discursivas o pragmáticas. Existe la necesidad de incorporar otras dimensiones, que aúnen las características del texto como mensaje a su estructura textual y su función social y comunicativa.

En Fernández Toledo (2000) se ahonda, entre otras cosas, en la relación entre el uso consciente de estrategias y la comprensión de géneros en inglés como LE. Específicamente se intentaba comprobar el efecto en la comprensión lectora del comportamiento estratégico (posible transferencia de la L1, uso de estrategias vinculadas a un procesamiento de tipo descendente, como las mencionadas, y otras más relacionadas con un acercamiento ascendente a los textos, etc.) y de aspectos relativos al género como unidad discursiva. En este trabajo 
se distingue entre estrategia estructural, relativa a la organización secuencial del texto (descripción, evaluación, etc.) de estrategia retórica, consistente en la identificación de la función(-es) pragmática(-s) del texto (convencer, informar, entretener...).

Los sujetos eran cincuenta sujetos de entre los alumnos de tercer curso de Biblioteconomía y Documentación (unos doscientos), que habían cursado inglés durante al menos tres años.

Como primer objetivo se planteó el comprobar si el uso de estrategias de lectura es similar para los sujetos en su lengua materna y en la LE, partiendo de la hipótesis, apuntada anteriormente por otros autores (Bossers, 1991; Raymond, 1993) de que existe transferencia de las destrezas lectoras (incluyendo el componente estratégico) de una lengua a otra. Para ello se utilizó un cuestionario adaptado del utilizado por Raymond (1993), en el que los hablantes contestaron sobre su propio comportamiento estratégico (véase apéndice).

Un segundo objetivo más específico era comprobar el efecto del uso de las estrategias retórica y estructural en la comprensión de los géneros en la L2, así como de su macroestructura, corroborando resultados anteriores (Lahuerta, 1994; Raymond, 1993). Por último, se intentó averiguar hasta qué punto el uso de estas dos estrategias iba unido, y si además iba acompañado del uso de otras estrategias de tipo metacognitivo. En este caso las pruebas incluían el resumen (en español como L1) de varios géneros en inglés y un cuestionario sobre los parámetros socio- cognitivos inherentes a cada texto- género (emisor, receptor, contexto, objetivo textual y función comunicativa).

Los resultados muestran una tendencia a utilizar las mismas estrategias en los dos idiomas y con casi la misma frecuencia. Las estrategias menos usadas por los sujetos fueron la de "pasar de largo algún término o expresión desconocido" y la de "guiarse por el objetivo convencional del texto". En el primer caso, sin embargo, los sujetos utilizaron más la estrategia con textos en inglés, siendo la puntuación para el español menor (quizá porque en este idioma no necesitan tanto hacer uso de ella). En el caso de la estrategia $\mathrm{n}^{\circ} 3$ ("mantener en mente la información que se va leyendo"), la frecuencia fue ligeramente menor en inglés, aunque seguía siendo alta.

Que los resultados sean prácticamente iguales para casi todas las estrategias en ambos idiomas no significa que el uso de todas las estrategias se transfiriera de la L1 a la LE. También pudo haber ocurrido lo contrario: en el caso de la primera estrategia (pasar de largo términos o expresiones desconocidos) ésta se suele reforzar en el aula de idiomas, aunque también se hace hincapié en ella en asignaturas relacionadas con la lingüística documental. Solamente una estrategia aparece como más típica de la L2, la referente al uso del contexto para averiguar léxico desconocido, o sea, el uso de la inferencia léxica. No es raro que así sea, ya que lo que más parece distinguir la lectura en L1 de la lectura en la LE es la automatización, siendo la inferencia el recurso más deseable ante un déficit de vocabulario en la lengua meta.

La estrategia menos utilizada tanto en la L1 como en la LE fue la relacionada con la función comunicativa o pragmática del texto (la estrategia $n^{\circ} 12$ en el cuestionario). Sin embargo, el uso de esta estrategia podría haber incidido en la mayor comprensión de los géneros, tanto de su contenido textual como de su marco interaccional, según se desprende de algunas dependencias significativas halladas. Estos datos corroboran la necesidad de un entrenamiento en el aula encaminado al uso consciente de dicha estrategia.

El uso de las estrategias 10 (ajustar la lectura al tipo de texto), 11 (retórica) y 12 (estructural) aparece estrechamente ligado, por lo que el entrenamiento explícito en uno de estos procedimientos podría favorecer el asentamiento de los otros. 
El uso de la estrategia estructural y el de la estrategia relativa al tipo de lectura según el género aparecen estrechamente ligados al de la estrategia retórica, por lo que el entrenamiento explícito en uno de estos procedimientos podría reforzar el de los otros. Esto concuerda con un estudio de Finkbeiner (1998) que demuestra cómo los estudiantes que han desarrollado un comportamiento estratégico en la lectura no utilizan estrategias aisladas, sino combinaciones de éstas, dependiendo de diversos factores.

Un dato curioso, sin embargo, es el ofrecido por Lahuerta (1994) acerca del uso consciente de pistas estructurales: sólo cuando hay consciencia por parte del sujeto acerca del beneficio de este uso, éste se traduce en una mejor comprensión. Esta constatación nos lleva de nuevo a subrayar la importancia del aspecto metacognitivo, tanto para la instrucción en esta estrategia como para la de las demás.

\section{Conclusiones}

Un factor crucial para la enseñanza de cualquier destreza, no sólo de la lectura, es el despertar en los alumnos la toma de conciencia sobre su propio aprendizaje, haciendo que se considere el responsable último de éste (Vygotsky, 1978; Wenden, 1998; Salataci y Akyel, 2002).

Se plantea como reto el averiguar hasta qué punto las estrategias enumeradas en los estudios revisados son generalizables a cualquier aprendiz; cómo enseñar de forma más efectiva estas estrategias y destrezas según el estilo de aprendizaje (Kirby, 1988); y hasta qué grado esta enseñanza debe ser explícita.

De cualquier modo, los hablantes de una segunda lengua o de una lengua extranjera parecen beneficiarse de un tratamiento contrastivo en el aprendizaje, en este caso de la comprensión lectora, de tal modo que parece ser de gran efectividad el sacar el máximo provecho de las relaciones entre lengua materna y lengua meta de manera explícita, algo que cuando se ha hecho en el aula de idiomas, no ha sido sin cierta culpabilidad o sensación de poca ortodoxia. Quizá sea necesaria cierta flexibilidad en estos temas, en aras de sacar el máximo provecho del conocimiento que nuestros alumnos, especialmente los universitarios, poseen desde antes de llegar a nuestras aulas.

Los estudios sobre estrategias estructurales y discursivas conforman un campo por explorar más a fondo, pero algunas de las conclusiones derivadas de los estudios reseñados, por corroborar en futuros trabajos, apuntan, por un lado, al beneficio de la instrucción explícita sobre la organización textual y del uso de estrategias para su detección. Esto es especialmente útil para los alumnos universitarios que han de procesar textos en inglés como lengua extranjera para la consecución de sus proyectos, ya que son estos textos (expositivos, argumentativos..) los que pueden presentar una estructura organizativa más compleja, siendo la instrucción explícita especialmente beneficiosa en estos casos.

Más importante si cabe es el uso de la estrategia discursiva, o lo que es lo mismo, la detección de la función(-es) pragmática(-s) inherente(-s) a cualquier texto como género. El beneficio de la instrucción explícita sobre su uso, por corroborar en futuros trabajos, afecta además positivamente al uso de otras estrategias como la estructural. Por tanto, y en tercer lugar, si el uso explícito de ciertas estrategias favorece un comportamiento estratégico general, la comprensión lectora se verá positivamente afectada, no sólo en la lengua meta, sino 
también en la propia del sujeto, si trabajamos en el aula las características genéricas de los textos (parámetros socioculturales inherentes, incluyendo el objetivo pragmático) y la forma de reconocerlas previamente a la lectura y durante ésta. El facilitar un comportamiento estratégico a la hora de leer textos en la lengua meta es sin duda beneficioso para nuestros alumnos, que posiblemente trasvasarán este comportamiento a nuevos textos, nuevos géneros y en distintas situaciones lingüísticas y comunicativas.

\section{REFERENCIAS}

Alderson, C. y Urquhart, A. H. (1988). "This test is unfair: I'm not an economist", en P.L. Carrell, J. Devine y D. Eskey (eds.), Interactive approaches to second language reading. Cambridge: Cambridge University Press, 168-182.

Bimmel, P., Van den Bergh, H. y Oostdam, R. (2001). "Effects of strategy training on reading comprehension in first and foreign language", en European Journal of Psychology of Education, 16, 4: 509-529.

Bossers, B. (1991). "On thresholds, ceilings and short-circuits: The relation between L1 reading, L2 reading and L2 knowledge", en AILA Review, 8: 45-60.

Carrell, P. (1984). "The effects of rhetorical organization on ESL readers", en TESOL Quarterly, 18, 3: 441-469.

Carrell, P. (1985). "Facilitating ESL reading by teaching text structure", en TESOL Quarterly, 19, 4: 727-745.

Carrell, P. (1987). "Content and formal schemata in ESL reading", en TESOL Quarterly 21, 3: 461481.

Carrell, P. (1988). "Interactive text processing", en P.L. Carrell, J. Devine y D. Eskey (eds.), Interactive approaches to second language reading. Cambridge: Cambridge University Press, 239-259.

Carrell, P. (1989). "Metacognitive awareness and second language reading", en The Modern Language Journal, 73, 2: 121-133.

Carrell, P., Carson, J. y Zhe, D. (1993). "First and second language reading strategies: Evidence from cloze", en Reading in a Foreign Language, 10, 2: 953-965.

Clarke, M. (1988). "The short-circuit hypothesis of ESL Reading — or when the language competence interferes with reading performance", en P.L. Carrell, J. Devine y D. Eskey (eds.), Interactive approaches to second language reading. Cambridge: Cambridge University Press, 114124.

Cohen, A. (1991). "Strategies in second language learning: insights from research", en R. Phillipson, E. Kellerman, L. Selinker et. al. (eds.), Foreign/ second language pedagogy research. Clevedon: Multilingual Matters, 107-119.

Connor, U. (1984). "Recall of text: Differences between first and second language readers", en TESOL Quarterly 18, 2: 239-256.

Cooper, D. (1990). Cómo mejorar la comprensión lectora [traducción de Jaime Collyer del título original Improving reading comprehension, 1986], Madrid: Visor Distribuciones S.A.

Demel, M. C. (1990). "The relationship between overall reading comprehension and comprehension of correferential ties for second language learners of English", en TESOL Quarterly, 24, 2: 267-290.

Dole, J., Brown, K. y Thraten, W. (1996). "The effects of strategy instruction on the comprehension performance of at-risk students", en Reading Research Quarterly, 31, 1: 62-88. 
Dole, J., Duffy, G., Roehler, L. et al. (1991). "Moving from the old to the new: Research on reading comprehension instruction", en Review of Educational Research, 61: 239-264.

Eisterhold, C.J., Carrell, P.L. Silberstein, S. et al. (1990). "Reading- writing relationships in first and second language", en TESOL Quarterly, 24: 245-266.

Fernández Toledo, P. (2000). Conocimiento previo, esquemas de género y comprensión lectora en inglés como lengua extranjera. Murcia: Servicio de Publicaciones de la Universidad de Murcia.

Fernández Toledo, P. (2002-3). "Esquemas formales y comprensión de géneros en lenguas extranjeras: estudio sobre la comprensión de los géneros reseña y anuncio editorial", en Pragmalingüística 10-11: 111-122.

Finkbeiner, C. (1998). "Are good readers also good strategists? The views of foreign language teachers", en Fremdsprachen Lehren und Lernen, 27: 180-203.

Fitzgerald, J. (1995). "English-as-a- second language learners' cognitive reading processes: A review of research in the United States", en Review of Educational Research, 65: 145-190.

Hammadou, J. (1991). "Interrelationships among prior knowledge, inference and language proficiency in foreign language reading", en The Modern Language Journal, 75: 27-38.

Hosenfeld, C. (1977). "A preliminary investigation of the reading strategies of succesful and unsuccesful second language learners", en System, 32: 110-123.

Jiménez, R. T. (1997). "The strategic reading abilities and potential of five low-literacy latina/o readers in middle school”, en Reading Research Quarterly, 32: 224-243.

Johnson, M. (1981). "Effects on reading comprehension of language complexity and cultural background of a text", en TESOL Quarterly, 15: 169-181.

Kern, R. (1989). "Second language reading strategy instruction: Its effects on comprehension and word inference ability", en The Modern Language Journal, 73: 135-149.

Kirby, J. (1988). "Style, strategy and skill in reading", en R. Schmeck (ed.), Learning strategies and learning styles. Londres: Plenum Press: 229-274.

Kitao, K., Yamamoto, M., Kitao, K. et. al. (1990). "Independent reading in English- Use of graded readers in the library English as a Second Language Corner", en Reading in a Foreign Language, 6, 2: 383-398.

Lahuerta, A. C. (1994). The use of text structure as a methodological tool to improve the reading comprehension of Spanish students of English at a University level. Tesis doctoral sin publicar. Universidad de Oviedo.

Lee, J. (1986). "On the use of the recall task to measure L2 reading comprehension", en Studies in Second Language Acquisition , 8: 201-212.

Lee, J. y Riley, G. (1990). "The effect of rhetorically- oriented frameworks in the recall of two structurally different expository texts", en Studies in Second Language Acquisition, 12: 2541.

León, J.A. (1991). "Intervención en estrategias de comprensión: un modelo basado en el conocimiento y aplicación de la estructura del texto", en Infancia y Aprendizaje, 56: 77-91.

Levine, A. y Reves, T. (1994). "The four- dimension model: Interaction of schemata in the process of reading comprehension", en TESL Canada Journal, 11, 2: 71-84.

Manchón Ruiz, R., Murphy, E. y Orts Llopis, M.A. (1993).“"Estrategias de inferencia y comprensión lectora en una L2”, en R.M. Manchón Ruiz y A. Bruton (eds.), Serie sobre estrategias de aprendizaje 1, Sevilla: Sand, 49-56.

McDonough, S. (1995). Strategy and skill in learning a foreign language. London: Edward Arnold. Meyer, B. (1975). The organization of prose and its effects on memory. Amsterdam: North Holland. 
Meyer, B. (1977). "The structure of prose: Effects on learning and memory and implications for educational practice", en R.C. Anderson, R.J. Spiro y W.E. Montague (eds.), Schooling and the acquisition of knowledge, Hillsdale (N.J.): Lawrence Earlbaum Associates.

Meyer, B. y Grice, E. (1982). "The interaction of reader strategies and the organization of text", en Text 2, 1-3: $155-192$.

Meyer, B. y Penland, J.M. (1982). "Effects of text structure on use of cognitive capacity during reading", en Journal of Educational Psychology 74, 1: 51-61.

Oláh, E. (1984). "How special is special English?", en A.K Pugh y J.M. Ulijn (eds). Reading for professional purposes, Londres: Heinemann, 223-226.

Pearson Casanave, C. (1988). "Comprehension monitoring in ESL reading: A neglected essential", en TESOL Quarterly, 22, 2: 283-302.

Peretz, A.S. y Shoham, M. (1990). "Teaching reading comprehension in LSP", en Reading in a Foreign Language 7, 1: 447-456.

Poggioli, L. (1991). "Investigación en la lectura: antecedentes y tendencias actuales", en A. Puente (ed.), Comprensión de la lectura y acción docente. Madrid: Pirámide; Salamanca: Fundación Germán Sánchez Ruipérez, 365-396.

Raymond, P. (1993). "The effects of structure strategy training on the recall of expository prose for university students reading French as a second language", en The Modern Language Journal , 77: 445-458.

Ridgway, T. (1994). Conocimiento previo y presentado por el texto en la lectura de una lengua extranjera. Tesis Doctoral sin publicar, Universidad de Sevilla.

Ríos Cabrera, P. (1991). "Metacognición y comprensión de la lectura." Comprensión de la lectura y acción docente. En A. Puente (ed.), Comprensión de la lectura y acción docente. Madrid: Pirámide; Salamanca: Fundación Germán Sánchez Ruipérez, 275-298.

Salager-Meyer, F. (1991). "Reading expository prose at the post-secondary level. The influence of textual variables on L2 reading comprehension (a genre-based approach)", en Reading in a Foreign Language, 8, 1: 645-662.

Salataci, R. y Akyel, A. (2002). "Possible Effects of Strategy Instruction on L1 and L2 Reading", en Reading in a Foreign Language 14, 1 (edición electrónica).

Tao, L. y Healy, A.F. (1996). "Cognitive strategies in discourse processing: A comparison of Chinese and English speakers", en Journal of Psycholinguistic Research, 25: 597-613.

Van Dijk, T. y Kintsch, W. (1983). Strategies of discourse comprehension. New York: Academic Press.

Vygotsky, L. S.(1978). Mind in society. The development of higher psychological processes. Cambridge (Mass.): Cambridge University Press.

Wenden, A. (1998). "Metacognitive knowledge and language learning", en Applied Linguistics, 19: 515-537.

Zuck, L.V. y Zuck, J.G. (1984). “The main idea: specialist and non-specialist judgements”, en A.K. Pugh y J.M. Ulijn (eds.), Reading for professional purposes, Londres: Heinemann, 130135 . 


\section{APÉNDICE \\ CUESTIONARIO SOBRE USO DE ESTRATEGIAS DE LECTURA EN INGLÉS \\ Y ESPAÑOL (adaptado de Raymond, 1993)}

Indica con qué frecuencia realizas las siguientes acciones cuando lees en tu lengua y cuando lees en inglés:

(S: siempre; AM: a menudo; AV: algunas veces; $\mathbf{C N}$ : casi nunca; N: nunca ; ESP: en español; ING: en inglés)

\begin{tabular}{|c|c|c|c|c|c|c|c|c|c|c|}
\hline & \multicolumn{2}{|c|}{$\overline{\mathrm{S}}$} & \multicolumn{2}{|c|}{$\overline{\mathbf{A M}}$} & \multicolumn{2}{|c|}{$\overline{A V}$} & \multicolumn{2}{|c|}{$\overline{C N}$} & \multicolumn{2}{|c|}{$\overline{\mathbf{N}}$} \\
\hline & Esp & Ing & Esp & Ing & Esp & Ing & Esp & Ing & Esp & Ing \\
\hline $\begin{array}{l}\text { 1. ¿Examinas las } \\
\text { ilustraciones u otros } \\
\text { elementos que } \\
\text { acompañan a los } \\
\text { textos? }\end{array}$ & & & & & & & & & & \\
\hline $\begin{array}{ll}2 . & \text { ¿Lees el título para } \\
& \text { deducir el tema del } \\
& \text { texto? }\end{array}$ & & & & & & & & & & \\
\hline $\begin{array}{l}\text { 3. ¿Mantienes en mente } \\
\text { la información que vas } \\
\text { leyendo? }\end{array}$ & & & & & & & & & & \\
\hline $\begin{array}{l}\text { 4. ¿Averiguas con ayuda } \\
\text { del contexto el sdo. de } \\
\text { términos } \\
\text { desconocidos? }\end{array}$ & & & & & & & & & & \\
\hline $\begin{array}{ll}\text { 5. ¿Utilizas tu } \\
\text { conocimiento } \\
\text { gramatical para } \\
\text { averiguar el sdo.de } \\
\text { palabras } \\
\text { desconocidas? } \\
\end{array}$ & & & & & & & & & & \\
\hline $\begin{array}{l}\text { 6. ¿Sueles reconocer las } \\
\text { palabras con forma } \\
\text { similar a las que } \\
\text { conoces en otras } \\
\text { lenguas? }\end{array}$ & & & & & & & & & & \\
\hline $\begin{array}{l}\text { 7. ¿Pasas de largo algún } \\
\text { término o expresión } \\
\text { que no conozcas? }\end{array}$ & & & & & & & & & & \\
\hline $\begin{array}{ll}\text { 8. ¿Utilizas tu } \\
\text { conocimiento del tema } \\
\text { para comprender la } \\
\text { información? }\end{array}$ & & & & & & & & & & \\
\hline
\end{tabular}




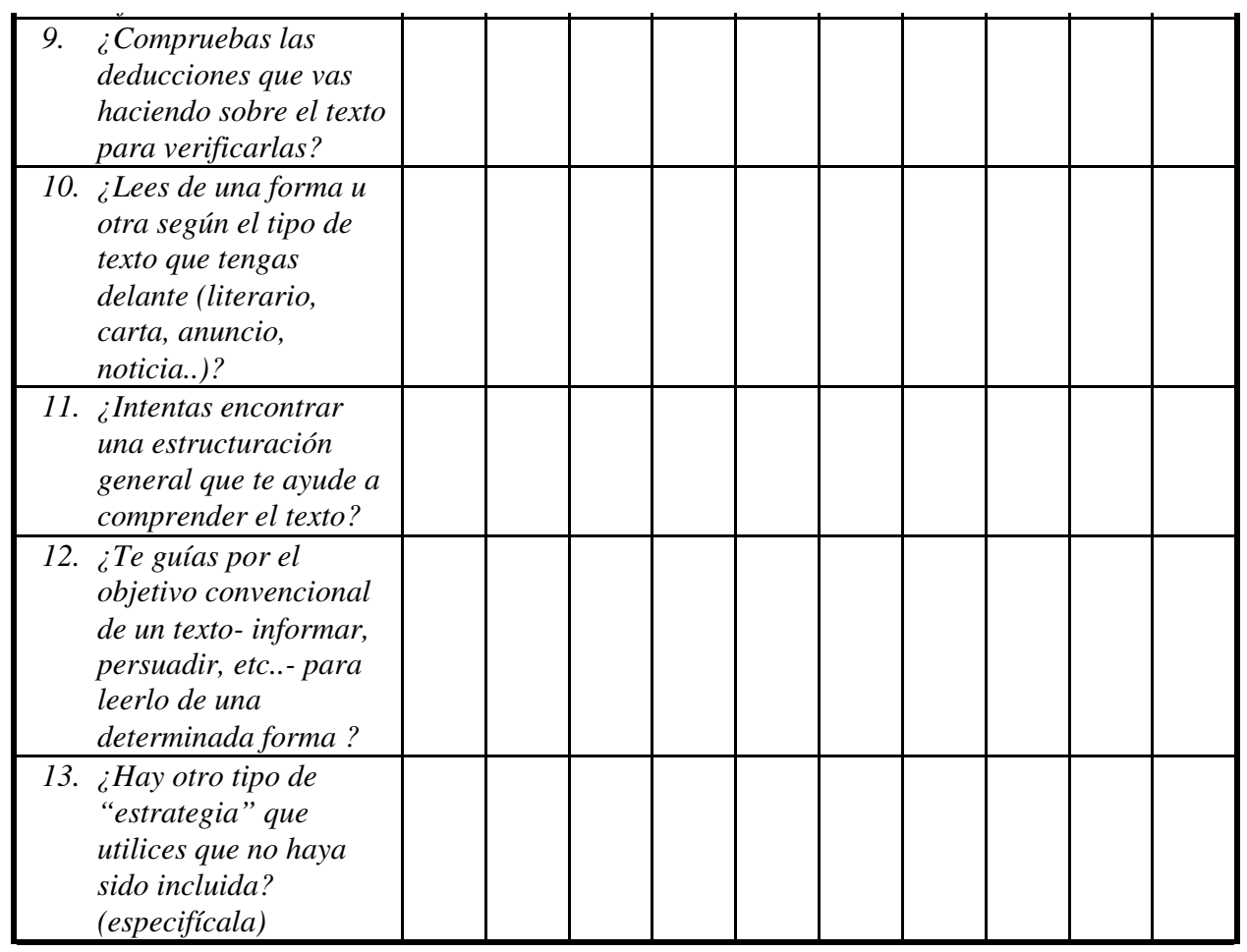

